\title{
Revisiting of Benzophenone Ketyl Still: Use of a Sodium Dispersion for the Preparation of Anhydrous Solvents
}

\author{
Ryo Inoue, ${ }^{\dagger}$ Mana Yamaguchi, ${ }^{\dagger}$ Yoshiaki Murakami, $^{\ddagger}$ Kentaro Okano, $^{\dagger}$ and Atsunori Mori ${ }^{*},{ }^{\dagger}$ \\ ${ }^{\dagger}$ Department of Chemical Science and Engineering, Kobe University, 1-1 Rokkodai, Nada, Kobe 657-8501, Japan \\ ${ }^{\ddagger}$ New Business Promotion Department, Kobelco Eco-Solutions Co. Ltd., 4-78-1 Wakinohama-cho, Chuo-ku, Kobe 651-0072, Japan
}

\section{Supporting Information}

ABSTRACT: A facile generation of organic solvents of anhydrous grade can be performed by distillation from sodium-benzophenone ketyl, which is prepared from commercial sodium dispersion and benzophenone. The distilled tetrahydrofuran, diethyl ether, hexane, 1,2-dimethoxyethane, and 1,4-dioxane with water contents less than $10 \mathrm{ppm}$ were obtained by a simple protocol. Safe treatment of the distillation residue also was simply achieved by the addition of methanol. The protocol suits for providing a small amount of an anhydrous solvent at the laboratory scale.

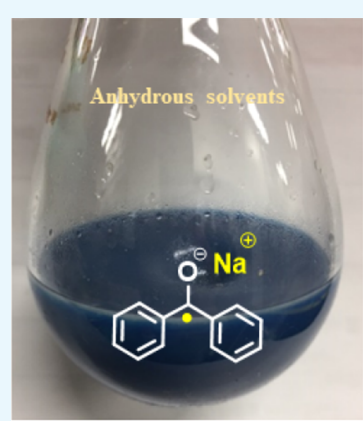

Sodium dispersion

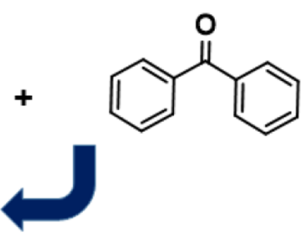

$\mathrm{THF}, \mathrm{Et}_{2} \mathrm{O}$, hexane, DME, 1,4-dioxane

\section{INTRODUCTION}

Use of an anhydrous organic solvent in a laboratory scale is an essential issue in modern organic synthesis. ${ }^{1}$ A wide range of organic and organometallic reactions particularly in transitionmetal catalysis and carbanion chemistry are carried out in an inert atmosphere, and therefore, the reaction should be performed with anhydrous solvents. ${ }^{2}$ Dehydration of solvents is carried out by treatment with sodium-benzophenone ketyl prior to distillation, in which a sodium lump/wire has been employed. However, the protocol requires significant care for the safe operation because of the highly explosive characteristics of the concentrated distillation residue to exposure to air as well as disposal of unreacted sodium metal after quenching with protic solvents such as alcohols and water. ${ }^{3}$ A new protocol for the dehydration of solvents to pass through alumina and copper columns developed by Grubbs was shown to produce extremely anhydrous solvents by a simple procedure, ${ }^{4}$ which completely replaced the conventional distillation protocol employing sodium-benzophenone ketyl. ${ }^{3}$ However, the system still involves difficulties concerning the cost of the dehydration apparatus as well as space limitation by increasing the kind of anhydrous solvents in a laboratory. Accordingly, on-demand construction of an additional distillation apparatus of benzophenone ketyl is still a choice to afford a dehydrated solvent if dehydration of the solvent can be performed in a safe manner. We herein disclose that the use of a sodium dispersion in a mineral oil ${ }^{5 a}$ in place of a sodium lump for the preparation of the benzophenone ketyl radical in a distillation flask provides several anhydrous solvents and treatment of the distillation residue by alcohol followed by water can be performed safely. Sodium dispersed in a mineral oil is remarkably stabilized to exposure to moisture in the air; however, the increased surface area improves the reactivity toward heterogeneous conditions compared with the use of a sodium lump. Indeed, a sodium dispersion has been developed for the dechlorinative degradation of polychlorinated biphenyls $(\mathrm{PCBs})^{5 \mathrm{~b}, \mathrm{c}}$ Several applications of a sodium dispersion to synthetic organic reactions have also been recently reported. ${ }^{6}$

\section{RESULTS AND DISCUSSION}

We first studied the dehydration of tetrahydrofuran (THF), which is widely employed in the reactions of anhydrous conditions in organic synthesis. The distillation vessel for THF was prepared by dissolving benzophenone $(1 \mathrm{mg} / \mathrm{mL})$ in a two-necked flask equipped with a distillation head as shown in Figure 1a. A sodium dispersion $(25 \% \mathrm{Na} \text { in mineral oil, w/w })^{a}$ was then added to the solution until the color of the mixture turned dark blue (Figure 1b). THF was then collected to the distillation head by heating the mixture at $85^{\circ} \mathrm{C}$ at an ambient pressure. Measurement of contents of water was carried out by a Karl-Fischer titration apparatus to reveal 7.23 ppm.? Although attempted dehydration employing 10 times larger amount of the sodium lump and benzophenone was also carried out, the mixture in the distillation vessel did not show any color change by the formation of ketyl at all. ${ }^{b}$ The result shows that a considerably larger amount of sodium and benzophenone is necessary for the dehydration in the use of the sodium lump. Thus, the use of a much smaller amount of the sodium dispersion effectively improved the dehydration efficiency because of its larger surface area. Distillation from the benzophenone ketyl/sodium dispersion of a higher content

Received: July 19, 2018

Accepted: September 24, 2018

Published: October 5, 2018 
(a)

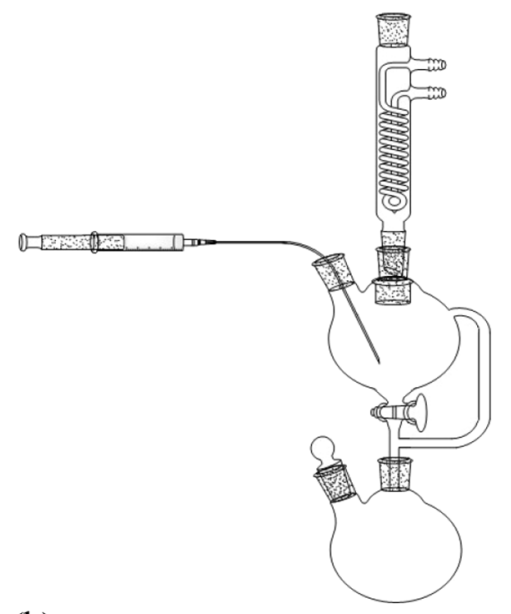

(b)

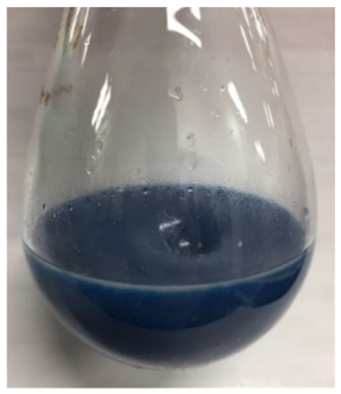

Figure 1. Distillation of the anhydrous solvent from sodium dispersion/benzophenone ketyl: (a) distillation apparatus and (b) sodium/benzophenone ketyl by the addition of the sodium dispersion and benzophenone into THF.

of sodium in mineral oil $(40 \%, \mathrm{w} / \mathrm{w})$ under similar conditions (normalized by the $\mathrm{Na}$ amount) showed a slightly inferior dehydration performance $(50 \mathrm{w} / \mathrm{w} \%$ in mineral oil: 17.34 $\mathrm{ppm})$. Although commercially available sodium sand (25-35 $\mathrm{w} / \mathrm{w} \%$ in paraffin) was also examined for the dehydration of THF, little color change by the formation of benzophenone ketyl was observed. Several organic solvents such as hexane, diethyl ether, 1,2-dimethoxyethane (DME), and 1,4-dioxane were dehydrated in a similar manner to afford the corresponding anhydrous solvents with water contents of $<10 \mathrm{ppm}$ as summarized in Table 1 . Contamination of the mineral oil of the sodium dispersion in the distilled solvent was hardly observed by ${ }^{1} \mathrm{H}$ NMR and gas chromatography (GC) analysis. $^{c}$

Because of a large volume of manipulating infrastructure of the Grubbs' column, ${ }^{4}$ a wide range of solvents for a permanent setup in a small-scale laboratory might be limited, despite the requirement of solvent diversity to be screened for the purpose of finding the most suitable conditions, which frequently occurs in the research of organic chemistry. Various solvents of the anhydrous grade are supplied commercially as $100 \mathrm{~mL}$ to 4 $\mathrm{L}$ in sealed bottles. The use of such dehydrated solvents could be highly effective when these solvents are consumed immediately once opened. However, the quality of the solvent as an anhydrous grade gradually becomes inferior after the bottle is frequently used by transferring to a syringe or a reaction vessel with a needle through the sealed rubber septum. Nevertheless, only a small quantity of an anhydrous solvent of $1-10 \mathrm{~mL}$ scale occurs frequently in the laboratory. A small size of the distillation apparatus for dehydration can be
Table 1. Distillation of Solvents from Sodium Dispersion/ Benzophenone Ketyl ${ }^{a}$

\begin{tabular}{lccc}
\multicolumn{1}{c}{ solvent } & $\begin{array}{c}\text { bath temp } \\
\left({ }^{\circ} \mathrm{C}\right)\end{array}$ & $\begin{array}{c}\text { boiling point } \\
\left({ }^{\circ} \mathrm{C}\right)^{b}\end{array}$ & $\begin{array}{c}\text { water contents } \\
(\mathrm{ppm})^{c, d}\end{array}$ \\
THF & 85 & 66 & $7.23(31.80)$ \\
THF & & & $17.34^{e}$ \\
diethyl ether & 40 & 35 & $6.84(123.0)$ \\
hexane & 90 & 69 & $3.80(33.06)$ \\
DME $^{f}$ & 110 & 82 & $6.82(156.8)$ \\
1,4-dioxane & 140 & 101 & $5.74(60.47)$
\end{tabular}

${ }^{a}$ Distillation from the sodium dispersion $(70 \mathrm{mg})$ and benzophenone $(50 \mathrm{mg})$ in $50 \mathrm{~mL}$ of the solvent. ${ }^{b}$ Data from suppliers. ${ }^{c}$ Measured by Karl-Fischer titration. ${ }^{d}$ In parentheses, water contents before distillation. ${ }^{e}$ Sodium dispersion of $40 \mathrm{w} / \mathrm{w} \%$ in mineral oil was employed. ${ }^{f}$ 1,2-Dimethoxyethane.

set in a corner of the laboratory bench, which is readily straightened once distillation is complete. The glassware apparatus can also be switched easily on the use of a different solvent.

It should be pointed out that, after collecting the solvent, visible aggregation of sodium metal was hardly observed in the concentrated residue composed of THF, sodium dispersion, and mineral oil. [Caution! The authors do not recommend further concentration of the distillation flask into almost dryness because safety to the explosive chain reaction of THF radical with oxygen has not been guaranteed to date.] The system was cooled to room temperature and the addition of methanol to the residue dropwise immediately showed the disappearance of the blue color of the ketyl radical to light yellow (Figure 2). ${ }^{d}$ Comparing with the post-treatment of the

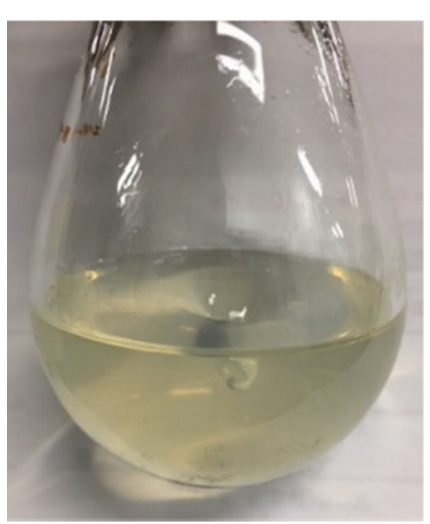

Figure 2. THF solution of benzophenone/ketyl from the sodium dispersion after quenching by methanol.

still of benzophenone ketyl employing a sodium lump, which generally requires the use of 2-propanol carefully for safety, it is remarkable that sodium dispersion can be easily quenched directly with methanol in a well-ventilated hood. Addition of the mixture into a large quantity of water resulted in phase separation and both phases thus separated were disposed safely.

\section{CONCLUSIONS}

In short, distillation generating an anhydrous solvent employing a sodium dispersion is quite simple employing switchable glassware. Several solvents of water contents of $<10 \mathrm{ppm}$ were obtained by simple distillation from benzophenone ketyl that 
was formed by the sodium dispersion. Quenching of the distillation residue can also be performed safely by the addition of methanol to result in the immediate disappearance of the blue color of benzophenone ketyl. Permanent setup of the distillation apparatus is not necessary on the laboratory bench. The protocol shows facile formation of a wide range of anhydrous solvents and therefore suits to experiments in a small laboratory scale (up to ca. $10 \mathrm{~mL}$ use) when a commercially available dehydrated solvent cannot be consumed within a short term to result in frequent transfer of the solvent with a needle through the sealed rubber septum.

\section{EXPERIMENTAL SECTION}

General. Solvents were purchased as an anhydrous grade from Kanto Chemical Co. Ltd., Nacalai Tesque, Inc., or Wako Pure Chemicals Co. Ltd. The sodium dispersion was employed as $25 \% \mathrm{Na}$ in mineral oil $(\mathrm{w} / \mathrm{w})^{a}$ or $50 \%(\mathrm{w} / \mathrm{w})$ available from Strem Chemicals Inc. The bottle was shaken vigorously prior to the use. Sodium sand $(25-35 \mathrm{w} / \mathrm{w} \%$ in paraffin) was purchased from Sigma-Aldrich Co. Ltd. Benzophenone from Sigma-Aldrich Co. Ltd. was used as purchased. Titration of water contents was performed with a Karl-Fischer titration apparatus (CA-21 Mitsubishi Chemicals Co. Ltd.). ${ }^{1} \mathrm{H}$ NMR $(400 \mathrm{MHz})$ spectra were measured on a JEOL ECZ spectrometer in $\mathrm{CDCl}_{3}$. GC analysis was carried out with Agilent7890A at Nittetsu-Sumikin Technologies Co. Ltd. (Himeji, Hyogo, Japan) with HP-5 ms $(0.32 \mathrm{~mm} \times 30 \mathrm{~m})$ using helium as a carrier gas at the flow rate of $2-4 \mathrm{~mL} / \mathrm{min}$ at the column temperature of $35-320{ }^{\circ} \mathrm{C}$ using a flame ionization detector detector.

General Procedure for the Distillation of the Solvent from Sodium Dispersion/Benzophenone Ketyl. To a 200 $\mathrm{mL}$ two-necked round-bottomed flask equipped with a magnetic stirring bar, distillation head, and nitrogen inlet was added $50 \mathrm{~mL}$ of THF, whose water content was $31.80 \mathrm{ppm}$. The flask was immersed in an oil bath. Benzophenone $(50 \mathrm{mg}$ ) was added to into THF to form a clear colorless solution to which the sodium dispersion $(70 \mathrm{mg})$ was added dropwise to observe the immediate color change to dark blue. The solution was refluxed for $2 \mathrm{~h}$ and then collected in the distillation head. The obtained dehydrated THF was taken to a syringe and subjected to Karl-Fischer titration to observe $7.23 \mathrm{ppm}$ of water contents. Dehydration of other solvents was carried out in a similar manner to show water contents less than $20 \mathrm{ppm}$ (see Table 1). Quenching of the distillation residue by methanol was carried out as follows: after cooling the distillation residue to room temperature, the flask was transferred to a well-ventilated hood. Then, $5 \mathrm{~mL}$ of methanol was added dropwise under a nitrogen atmosphere. The color of the mixture immediately changed to light yellow. Water (50 $\mathrm{mL}$ ) was added to the mixture to observe separation into organic and aqueous phases.

\section{ASSOCIATED CONTENT}

\section{S Supporting Information}

The Supporting Information is available free of charge on the ACS Publications website at DOI: 10.1021/acsomega.8b01707.

${ }^{1} \mathrm{H}$ NMR spectra and GC profiles of the distilled solvent and the mineral oil employed for the generation of the sodium dispersion (PDF)

\section{AUTHOR INFORMATION}

\section{Corresponding Author}

*E-mail: amori@kobe-u.ac.jp.

ORCID

Kentaro Okano: 0000-0003-2029-8505

Atsunori Mori: 0000-0002-1163-264X

Notes

The authors declare no competing financial interest.

\section{ACKNOWLEDGMENTS}

The authors thank Professor Tatsuo Maruyama of Kobe University for valuable suggestion and instruction on the use of Karl-Fischer titration.

\section{ADDITIONAL NOTES}

${ }^{a}$ Sodium dispersion is supplied commercially as a dispersion in a mineral oil by Kobelco Eco Solution. Co. Ltd.

${ }^{b}$ Water contents of THF distilled from the sodium (lump) benzophenone ketyl is reported to show $43.4 \pm 0.7 \mathrm{ppm}$ after refluxing for $48 \mathrm{~h}$. See ref $4 \mathrm{~b}$.

${ }^{c}$ Distilled THF was subjected to ${ }^{1} \mathrm{H}$ NMR and GC analyses and compared with that of authentic mineral oil. See Supporting Information.

$d_{\text {The color of the ketyl radical was also confirmed to disappear }}$ upon exposure of the cooled residue to air within a short period.

\section{REFERENCES}

(1) Armarego, W. L. F.; Chai, C. L. L. Purification of Laboratory Chemicals, 6th ed.; Elsevier: Amsterdam, 2009.

(2) (a) Experimental Organometallic Chemistry; Wayda, A. L., Darensbourg, M. Y., Eds.; ACS Symposium Series; American Chemical Society: Washington, DC, 1987; Vol. 357. (b) Errington, R. J. Advanced Practical Inorganic and Metalorganic Chemistry, 1st ed.; Blacky Academic \& Professional: London, 1997.

(3) (a) Leorand, J.; Lygo, B.; Procter, G. Advanced Practical Organic Chemistry, 3rd ed.; CRC Press: Boca Raton, 2013. (b) Cournoyer, M. E.; Dare, J. H. The Use of Alternative Solvent Purification Techniques. Chem. Health Saf. 2003, 10, 15-18.

(4) (a) Pangborn, A. B.; Giardello, M. A.; Grubbs, R. H.; Rosen, R. K.; Timmers, F. J. Safe and Convenient Procedure for Solvent Purification. Organometallics 1996, 15, 1518-1520. (b) Williams, D. B. G.; Lawton, M. Drying of Organic Solvents: Quantitative Evaluation of the Efficiency of Several Desiccants. J. Org. Chem. 2010, 75, 8351-8354.

(5) (a) Ariizumi, A.; Otsuka, T.; Kamiyama, M.; Hosomi, M. Dechlorination and Decomposition Behavior of PCBs by the Sodium Dispersion Process. J. Environ. Chem. 1997, 7, 793-799. (b) Noma, Y.; Mitsuhara, Y.; Matsuyama, K.; Sakai, S.-i. Pathways and Products of the Degradation of PCBs by the Sodium Dispersion Method. Chemosphere 2007, 68, 871-879. (c) Huang, H.; Kobayashi, N.; Hasatani, M.; Matsuyama, K.; Sasaki, T. Reaction Kinetics Analysis of the Dechlorination Process of PCBs by Sodium Dispersion Process. Chem. Eng. Sci. 2007, 62, 5144-5149. (d) Murakami, Y. Jpn. Kokai Tokkyo Koho, JP 2006-340051. (e) Ware, M. L.; Argentine, M. D.; Rice, G. W. Potentiometric Determination of Halogen Content in Organic Compounds Using Dispersed Sodium Reduction. Anal. Chem. 1988, 60, 383-384. (f) Menville, R. L.; Parker, W. W. Determination of Organic Halides with Dispersed Sodium. Anal. Chem. 1959, 31, 1901-1902. (g) Nobis, J. F.; Moormeier, L. F.; Robinson, R. E. Organosodium Compounds for Preparation of Other Carbon-Metal Bonds. In Metal Organic Compound; American Chemical Society, 1959; pp 63-68.

(6) (a) Gissot, A.; Becht, J.-M.; Desmurs, J. R.; Pévère, V.; Wagner, A.; Mioskowski, C. Directed Ortho-Metalation, a New Insight into 
Organosodium Chemistry. Angew. Chem., Int. Ed. 2002, 41, 340.

(b) An, J.; Work, D. N.; Kenyon, C.; Procter, D. J. Evaluating a Sodium Dispersion Reagent for the Bouveault-Blanc Reduction of

Esters. J. Org. Chem. 2014, 79, 6743-6747. (c) Zhang, B.; Li, H.;

Ding, Y.; Yan, Y.; An, J. Reduction and Reductive Deuteration of Tertiary Amides Mediated by Sodium Dispersions with Distinct Proton Donor-Dependent Chemoselectivity. J. Org. Chem. 2018, 83, 6006-6014.

(7) (a) Fischer, K. Neues Verfahren Zur Maßanalytischen Bestimmung Des Wassergehaltes von Flüssigkeiten Und Festen Körpern. Angew. Chem. 1935, 48, 394-396. (b) Dantan, N.; Frenzel, W.; Küppers, S. Determination of Water Traces in Various Organic Solvents Using Karl Fischer Method under FIA Conditions. Talanta 2000, 52, 101-109. 